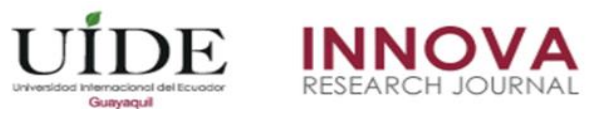

INNOVA Research Journal, ISSN 2477-9024

(Febrero, 2018). Vol. 3, No.2 pp. 27-38

DOI: https://doi.org/10.33890/innova.v3.n2.2018.626

URL: http://revistas.uide.edu.ec/index.php/innova/index

Correo: innova@uide.edu.ec

\title{
La caracterización glocal como estrategia comunitaria: Competencias pertinentes del gestor social del conocimiento
}

\section{The glocal characterization as a community strategy: Relevant competencies of the social knowledge manager}

Ericka Figueroa Martínez

María del Carmen Rivera Villalta

Lucrecia Resabala Manosalvas

Universidad de Guayaquil, Ecuador

Autor para correspondencia: ericka.figueroama@ug.edu.ec, maría.riverav@ug.edu.ec, lucrecia.resabalam@ug.edu.ec

Fecha de recepción: 27 de Julio 2017 - Fecha de aceptación: 15 de Enero de 2018

Resumen: En el presente proyecto, se definen los conocimientos previos que tiene el gestor social del conocimiento basado en la normativa de las Instituciones de Educación Superior, la representación de la misma en el contexto de la localidad como escenario pertinente que necesita conocer el docente-gestor, ya que el trabajo es in situ, vital para la coyuntura del trabajo que realiza en su acercamiento a la comunidad. La metodología es participativa, con un diseño bibliográfico. Los núcleos teóricos son: lineamientos para el diseño de proyectos de responsabilidad social, reglamentos de funciones de los gestores de Gestión Social del Conocimiento, gestión de recursos, instructivos para el manejo de matrices de informes y matrices de seguimiento y monitoreo de la implementación de los proyectos en las prácticas de vinculación con la colectividad. La perspectiva de las funciones del gestor se entrelazan con las funciones sustantivas como: Investigación, Gestión Social del Conocimiento y Posgrado. El objetivo, es caracterizar las funciones de seguimiento y monitoreo del gestor de proyectos de responsabilidad social como gestor de vinculación dentro de las funciones glocales siendo su perspectiva y proyección el efectuar un trabajo social relevante y participativo con la comunidad.

Palabras claves: responsabilidad social; gestión social; prácticas preprofesionales; gestión de recursos; vinculación con la comunidad

Abstract: In the present project, the previous knowledge of the social manager of knowledge based on the regulations of the Institutions of Higher Education is defined, the representation of the same in the context of the locality as a relevant scenario that the teacher-manager needs to know, since the work is in situ, vital for the conjuncture of the work that it carries out in its approach to the community. The methodology is participatory, with a bibliographic design. The theoretical nuclei are: guidelines for the design of projects of social responsibility, regulations of functions of the managers of Social Knowledge Management, resource management, instructions for the management of reporting matrices and monitoring matrices and monitoring of the implementation of the projects in the practices of connection with the community. The perspective of the functions of the manager are intertwined with the substantive functions such as: Research, Social Knowledge Management and Postgraduate. The objective is to characterize the monitoring and monitoring functions of the social responsibility project manager as a linkage manager within the glocal functions, its perspective and projection being to carry out a relevant and participatory social work with the community.

Key Words: social responsibility; social management; internships; resource management; community liaison 


\section{Introducción}

El presente proyecto se desarrolla en la Facultad de Filosofía, Letras y Ciencias con la revisión de documentos de autores probos que demuestran su experticia investigativa en el área de la Gestión Social del Conocimiento. El desarrollo del proyecto se enfocó en dos aristas: el reconocimiento de la estructura, base del proyecto que se desarrolla en nuestro claustro y la pertinencia del mismo en relación a las necesidades del constructo social.

Los indicadores que se desarrollan son las competencias sustantivas: investigación, diseño y ejecución de proyectos sociales y programas de posgrados, los programas que se desarrollan son de cohorte educativa y funcional con organizaciones del estado y privado.

El proceso vinculante tiene sus inicios en la investigación de las necesidades de las comunidades especialmente de grupos vulnerables de la zona de influencia de la Universidad de Guayaquil que requieran apoyo en los procesos educativos, de ahí surgen proyectos de responsabilidad social acorde a los perfiles de las carreras de la Facultad de Filosofía que respondan a las necesidades de las comunidades; paralelamente a esto se establecen convenios con aliados estratégicos, es decir instituciones cercanas a la comunidad o que tengan dentro de su misión ayudar a sectores vulnerables, estas son las que apoyan la gestión del proyecto.

Cabe destacar que este proceso se enmarca en lineamientos de las instituciones que rigen el desarrollo de la Educación Superior y con el aval de las autoridades de la Universidad de Guayaquil, pero no podemos descartar los acuerdos con la comunidad vinculante e instituciones con las que se firman los convenios, el fin último es que nuestros estudiantes pongan en práctica las habilidades adquiridas en su formación y adquieran desempeños beneficiando a grupos que están en estado de vulnerabilidad.

Los estudiantes adquieren experiencia, la Universidad cumple son su misión de ser una institución al servicio de la sociedad y se contribuye a mejorar una problemática desarrollando el sentido de responsabilidad social en todos los actores involucrados.

\section{Objetivos General}

Caracterizar la gestión del agente de vinculación dentro de las funciones glocales siendo su perspectiva y mística encaminado a la realización de un trabajo social relevante y participativo.

\section{Metodología}

Según Arnaz (2011) "La metodología participativa consiste en concebir en proyectos de cooperación para la comunidad donde se ejecuta, un desarrollo sostenible, protagonizado por la propia población en consonancia de la democracia participativa" (p.36)

A través de estos recursos educativos que brinda la Facultad de Filosofía, Letras y Ciencias de la Educación, promueve el desarrollo y el crecimiento de la población en la que se 
glocalizo la estructura comunitaria, pues está va adquiriendo protagonismo en la toma de decisiones y en la gestión de recursos.

Estos habitantes se convierten en actores determinantes de su propio desarrollo y se potencia la capacidad política y económica de toda la comunidad local, incluyendo los sectores sin poder y con mayores niveles de pobreza, vulnerabilidad y exclusión social.

\section{Desarrollo}

\section{Características del Gestor Social de Conocimiento}

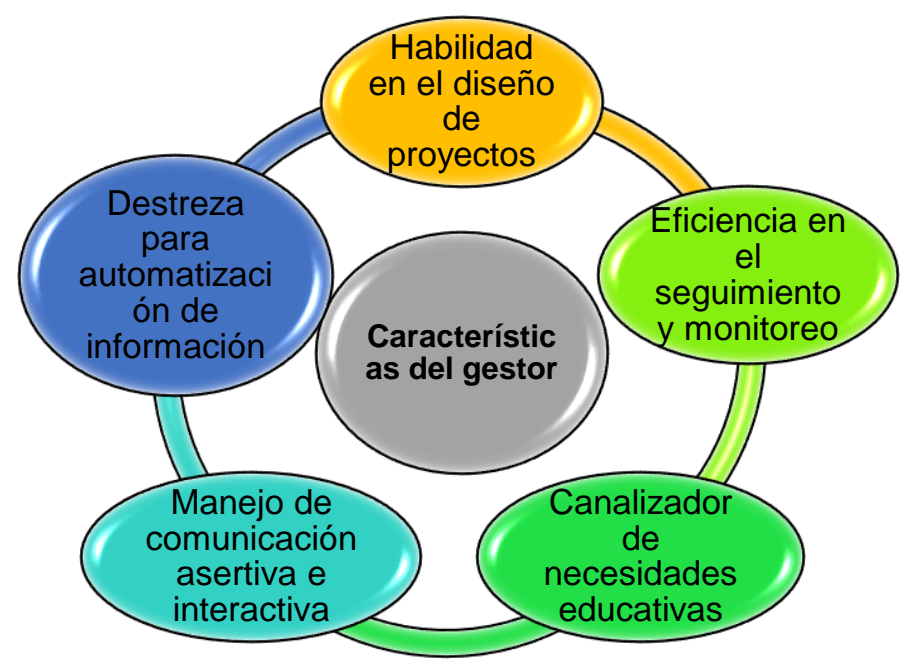

\section{El Plan Estratégico Institucional como agente objetivado de las premisas de la Gestión Social del Conocimiento.}

Actualmente todas las instituciones y gestiones deben estar relacionadas con un proceso de planificación para que las actividades estén planificadas y se prevea toda gestión, por ello se recomienda que para esta gestión se incluya o considere el Plan estratégico de la comunidad articulado al de la Universidad.

La importancia de incorporar esta planificación estratégica es que se ha convertido, en una rama de los negocios y en el desarrollo de proyectos educativos para conseguir los objetivos trazados por los componentes sociales, la misma que puede ser aplicada con éxito en diferentes escenarios, uno de ellos el que está en el contexto de las comunidades que buscan un desarrollo humano integral y el otro el que participa principalmente en la mejora de la calidad de vida.

Esta planificación debe estar direccionada a la investigación científica, tecnología, innovación y conocimiento científico; este vínculo con la sociedad debe tener proyección en los valores, necesidades y problemas sociales considerando que el presupuesto de los proyectos pueden se aportaciones gubernamentales o no gubernamentales.

Alineación del perfil de salida del estudiante con relación al proyecto de vinculación: 


\section{Saberes}

\section{Habilidades y destrezas}

\section{Perfil del egreso}

\section{Actitudes}

Realizado: MSc Ericka Figueroa Martínez y MSc. Lucrecia Resabala

Los programas de Gestión Social de Conocimiento se alinean a programas: Integración educativa en la diversidad, actividades, construcción transferencia, inclusión y ciudadanía, Buen Vivir y al modelo pedagógico de la Universidad de Guayaquil. El fin es mejorar el proceso formativo del estudiante no sólo en el área cognitiva y procedimental, sino el aspecto actitudinal, pues desarrollará empatía con su entorno social.

\section{Las fortalezas del gestor social del conocimiento y sus debilidades desde la glocalidad.}

Las fortalezas, tienen una conexión directa con el éxito del proceso de trabajo en la gestión social del conocimiento, su desarrollo permite el crecimiento del juicio gestacional del mismo, pero sobre todo las cualidades que tengan el docente-gestor y el reconocimiento territorial permitirán las metas in situ y en tiempo real, pero si el proyecto no aterriza en realidades existentes desde el estudio de las necesidades y la pertinencia, las debilidades en el cumplimiento del proyecto serán extremadamente superiores al objetivo del mismo, los componentes que se deben desarrollar después de este análisis es que los procesos deben cumplir las competencias genéricas básicas de formación social que indica la Universidad de Guayaquil y son: comunicación oral y escrita, resolución de conflictos, comunicación multilinguistica, respeto a la diversidad, liderazgo.

(Porter, 1998) "Las fortalezas y oportunidades son un conjunto, las capacidades que se manifiestan en el estudio tanto de los aspectos fuertes como débiles de las organizaciones..." (p.407)

El proceso de elección del personal llamado Supervisor o Gestor Social del Conocimiento debe tener ciertas características de competencias necesarias como: la interpretación de los componentes sociolingüísticos, argumentación comunicacional, la propuesta de estrategias de desarrollo comunitario, identificar los componentes lingüísticos.

\begin{tabular}{lllll}
\hline & \multicolumn{3}{c}{$\begin{array}{c}\text { Competencias de Formación Social } \\
\text { Desempeño }\end{array}$} & $\begin{array}{c}\text { Características de Gestor Social del } \\
\text { Conocimiento }\end{array}$ \\
\cline { 2 - 6 } $\begin{array}{l}\text { Comunicación oral } \\
\text { y escrita }\end{array}$ & $\begin{array}{l}\text {-Interpreta } \\
\text { componentes socio-lingüístico, histórico } \\
\text { y pragmático. }\end{array}$ & $\begin{array}{l}\text { Interpreta el desarrollo histórico-social del } \\
\text { constructo social. }\end{array}$ \\
& $\begin{array}{l}\text {-Argumenta } \\
\text { elementos comunicativos, habilidades y } \\
\text { destrezas. }\end{array}$ & $\begin{array}{l}\text { Identifica con conectores lingüísticos las } \\
\text { entorno educativo }\end{array}$ \\
\hline
\end{tabular}




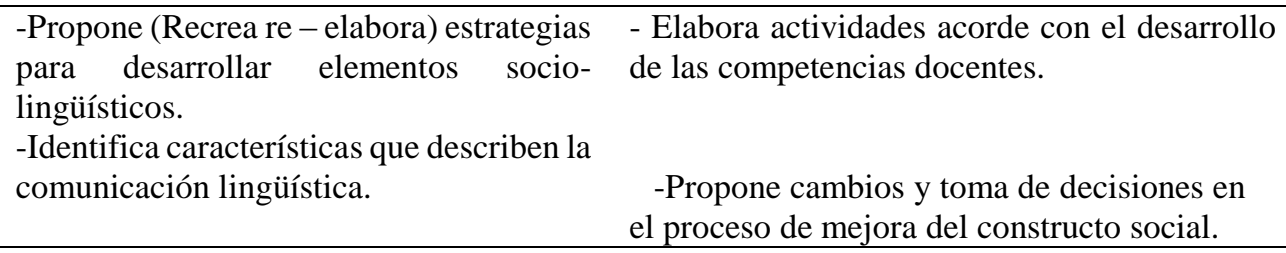

-Propone cambios y toma de decisiones en el proceso de mejora del constructo social.

\section{Fuente: (UG, Competencias básicas de la Universidad de Guayaquil, 2016)} Realizado por: MSc Ericka Figueroa Martínez

Estos procesos se desarrollan en el desarrollo de un proyecto que tiene aristas claras con un modelo de Marco Lógico, de investigación y desarrollo de actividades que motiven a la solución de problemas.

Como lo indica Alfredo García Pye en el libro Rol del Gestor Social del Conocimiento, Programa profesional de futuro "Hoy es el rol del gestor social es de bombero apagando incendios cuando en realidad debe ser un constructor de puentes articulados, de relación, un generador de confianza"

El Gestor Social de Conocimiento, debe crear espacios de trabajo y concienciación social es un ente con un vasto conocimiento en proyectos, presupuestos, el proceso de marco lógico, da resolución de conflictos y planteamiento de soluciones reales acorde al constructo social.

(Sodexo, 2015) "Un gestor que conoce las costumbres del entorno y es capaz de mimetizarse gracias a sus conocimientos generalizados de la región. Es un profesional que así como conoce al milímetro el funcionamiento de su empresa, conoce todo sobre sus stakeholderse incluso sobre el Estado, su forma de operar, trámites y permisos"

Este experto reconoce en el área educativa el proceso mentor del supervisor y los estudiantes en cuanto a la flexibilidad territorial sus vínculos se entrelazan en los proyectos establecidos con el fin de ayudar a la Comunidad Educativas ( Beneficiarios Directos e Indirectos ) en la Facultad de Filosofía, Letras y Ciencias de la Educación que son: Educación, Responsabilidad Social y Bibliotecología.

En la elaboración del escenario educativo, es necesario identificar el problema que va a solucionar el proyecto; determinando sus características más relevantes, detallando sus causas y repercusiones o efectos. En la elaboración del diagnóstico se deberá analizar la situación actual o existente del área de intervención del proyecto, considerando: localización, límites, población desagradada por sexo, etnia, edad, educación, salud, servicios básicos, viabilidad, entre otros datos relevantes.

(Ponce, 2006) Indica que la oportunidad es un desafío: “Las oportunidades constituyen aquellas fuerzas ambientales de carácter externo no controlables por la organización” (p.16)

La Gestión Social del Conocimiento, permite al docente-tutor reconocer las perspectivas del entorno social que encuentra anclado en la misión y visión de los proyectos que se desarrollan con los diferentes componentes y que tiene que ver con proyectos marco o específicos, los que tiene firmados la Universidad de Guayaquil, Facultad de Filosofía, Letras y 
Ciencias de la Educación. Esta oportunidad que tiene el docente que es Gestor o Supervisor le permite vivenciar con sus integrantes el desarrollo comunitario.

(Talancón, 2006) Indica que las premisas que desarrollan oportunidad son más factibles en el proyecto desde las oportunidades:

"Las oportunidades constituyen aquellas fuerzas ambientales de carácter externo no controlables por la organización, pero que representan elementos potenciales de crecimiento o mejoría. La oportunidad en el medio es un factor de gran importancia que permite de alguna manera moldear las estrategias de las organizaciones" (p.2)

Para el desarrollo de las Gestores Social del Conocimiento

\section{Técnicas participativas para revisar los proyectos de cohorte comunitaria}

\begin{tabular}{lll}
\multicolumn{1}{c}{ Autodiagnóstico } & \multicolumn{1}{c}{ Planificación } & Ejecución y seguimiento \\
\hline Transectos & Árbol de objetivos & Núcleos de intervención \\
Sociograma & Matrices reflexivas & Coherenciometro \\
Rol playing & Teatro-forum & \\
Línea de tiempo & Técnicas de priorización & \\
Mapas emocionales & Técnicas semáforo & \\
FODA & \\
Delphi & \\
Flujograma & & \\
Árbol de problemas & &
\end{tabular}

Fuente: (Internacional, 2016)

\section{Políticas de Gestión Social del Conocimiento como formas sustantiva}

$\begin{array}{ccc}\text { PREGUNTAS } & \text { RESPUESTAS } & \text { EVIDENCIAS } \\ \text { (CEAACES) } & \text { (UG) } & \text { (AREA/DPTO. UG) }\end{array}$

¿Cómo se garantiza que los
proyectos de vinculación
vigentes estén alineados a la
planificación estratégica y
operativa institucional, y
respondan a las necesidades
del entorno?

del entorno?
La estructura de los programas y proyectos, se encuentran alineados a la Planificación Estratégica Institucional, ya que el Plan Integrado de Vinculación con la Sociedad contempla el desarrollo y cumplimiento de sus objetivos. De igual forma los programas y proyectos son formulados en concordancia con los Dominios definidos por la UG, las líneas y sublíneas de Investigación, respondiendo a las necesidades del entorno al determinar la línea base de las comunidades y beneficiarios del proyecto, contrastándolos con los objetivos estratégicos nacionales del PNVB y sus agendas sectoriales y zonales.
1.4.1.1 - $2 \mathrm{a}$ Instructivo de responsabilidad social

1.4.1.1-2b Estructuras de Programas y proyectos.

1.4.1.1 - 2j Políticas Gestión Social del Conocimiento

Plan Integrado de Vinculación Plan Nacional del Buen Vivir Dominios PEDI (Plan Estratégico Institucional) 
Agendas Sectoriales y Zonales

¿De qué manera se integra a la vinculación, a la academia y a la investigación?

¿Cómo se asegura que la gestión de recursos para los proyectos de vinculación es suficiente y oportuna?
Los programas y proyectos de vinculación se integran por una parte a la Academia: con la atención de los problemas y necesidades identificadas en la comunidad a través de la ejecución de las prácticas pre profesiones, y de esta forma el estudiante con la guía del docente tutor, realiza el engranaje de la teoría y la práctica fortaleciendo sus conocimientos.

Por otra parte, los programas y proyectos de vinculación se articulan a la Investigación: ya que a) se identifican las necesidades o vacíos que pudiese existir en la comunidad para la resolución de $\mathrm{X}$ problema, b) se recopilan $\mathrm{y}$ formulan nuevos insumos, mismos que pueden servir de base para posibles investigaciones y nuevas teorías o metodologías, c) se sistematizan experiencias capaces de ser traducidas para conferencias, ponencias, y demás publicaciones.

A través de la planificación de los programas y proyectos, en los que se incluye el presupuesto de ejecución de los proyectos y estos se consolidan en la planificación operativa anual.

A través de matrices de monitoreo, que permite determinar el avance de los proyectos y el contraste con el cumplimiento de los objetivos y la planificación de los mismos; el impacto se lo determina en el informe final de ejecución del proyecto. 1.4.1.1-2b

Estructuras de

Programas y proyectos.

1.4.1.1 - 2j Políticas

Gestión Social del

Conocimiento

Instructivo de práctica preprofesional
1.4.1.1-4

Planificación

Operativa 2014

1.4.1.1-5

Planificación

Operativa 2015

1.4.1.2 - 13-14

Documentos que certifiquen la Ejecución 2014-2015

1.4.1.2 - 15

Planificación

presupuestaria 2014

1.4.1.2 - 16

Planificación

presupuestaria 2015

1.4.2.1 - 19

Normativa de vinculación 1.4.2.1 - 20-21

Informe de seguimiento 20142015

1.4.2.1 - 23

Programas y/o proyectos

Informe final de ejecución e impacto del proyecto

Fuente: (UG, 2016)

Según el objeto de estudio el conocimiento se debe saber contextualizar, globalizar, multidimensionar, es decir, debe ser complejo. 
En la vía (Morín, 2011) identifica el problema crucial de este tiempo como la necesidad de un pensamiento que pueda asumir el desafío "complejo de lo real, esto es, de captar las relaciones, interacciones e implicaciones mutuas, los fenómenos multidimensionales, las reflexiones solidarias y conflictivas a la vez (p.12)

(Bordieau, 2000) Indico en su informe

\begin{tabular}{ll}
\hline & Objetivar el hecho social \\
\hline & La práctica del medio y constructo \\
& La metodología y su proyección en el espacio social \\
Hechos objetivados & Las dimensiones desde su estado de pertinencia \\
& Sus adhesiones sociales \\
& Las categorías de análisis: edad, sexo, estrato social. \\
\hline
\end{tabular}

Para Bordieau y Morín indican en sus escritos la buena práctica del reconocimiento de los ambientes sociales que necesita una revisión del constructo social, generadora de espacios colaborativos en donde las categorías de análisis se revisan desde la necesidad que existe en esa población para realizar cualquier tipo de análisis.

\section{La necesidad del reconocimiento de la experiencia del entorno social}

El modelo de Gestión Universitaria realizado por SENESCYT con las universidades de excelencia donde se establece la interacción de los sistemas de formación, investigación, vinculación con la sociedad, y la gestión administrativa financiera, como marco de acción y coordinación de toda la universidad se desarrollan en dimensiones.

(Ramirez, 2012) Las dimensiones de la ciudadanía son:

Las dimensiones de la ciudadanía son:

El eje civil, que se expresa en los derechos de igualdad entre ciudadanos; política, que describe los derechos y obligaciones en el ejercicio del poder político al ciudadano; social, los derechos a condiciones materiales que atiendan las necesidades humanas; económica, que rige las relaciones de poder entre obreros y patrones; y la cultural, correspondiente al derecho de acceder a los bienes culturales. A las dimensiones de la ciudadanía, Ramírez Saiz atañe una serie de ejes estructurantes, los cuales otorgan instrumentalidad a la cualidad del ciudadano:

- El eje jurídico representado por la forma en que las instituciones reconocen al ciudadano

- El eje antropológico o cultural que se expresa en la percepción propia del carácter ciudadano y, por tanto, la pertenencia a una comunidad política.

- El eje de la práctica o agencia ciudadana, logrado mediante las formas colectivas para el ejercicio de derechos y responsabilidades.

- Y el eje institucional, que engloba a las instituciones que defienden o administran los derechos de los ciudadanos. 
El docente que fomenta el principio de Gestor Social del Conocimiento debe practicar estos ejes: jurídico, antropológico, de práctica, agencia ciudadana e institucional, pues defienden los derechos colectivos que se promulgan en la glocalidad en la que se encuentran la intervención comunitaria o reconocer el territorio.

\section{Prácticas Pre Profesionales y su prevalencia dentro del desarrollo holístico de la vinculación con la comunidad}

La vinculación con la colectividad, es uno de los procesos fundamentales que se instituyen en la universidad actual; pero esto no siempre se realiza de forma consciente y organizativa, por eso se debe desarrollar espacios en la comunidad que generen aspectos de tipo científico, humanístico, técnico, social y cultural.

Este proceso de articulación de las procesos educativos de los estudiantes universitarios a los procesos de desarrollo social llamados vinculación es un requisito para la titulación y es considerado una parte fundamental de las prácticas pre profesionales.

Las prácticas pre profesionales llamadas así tienen como objetivo articular lo teórico con la experiencia, es decir desarrollar proceso en los que el estudiante pueda poner en práctica los conocimientos, la vinculación es el contacto con la comunidad es afianzar los valores y el desarrollo de la vocación mediante la puesta en la acción los perfiles del egreso dependiendo de la espeicalidad.

"Indica que entre los procesos sustantivos de la universidad, para atender las demandas sociales, están la docencia o academia, investigación y la extensión, siendo estas tres instancias las que engloban el ser de las IES"

Según la extensión universitaria tiene como visión el papel debe jugar esta última en el desarrollo social y los contenidos articulación.

(Caicedo, 2014) "Resulta importante que los estudiantes en su formación pre profesional se vinculen con investigaciones y actividades que fortalezcan la función de la vinculación con la colectividad" (p.17)

\section{Modelo de Planificación Estratégica Comunitaria}

\begin{tabular}{ll}
\hline Equipo de trabajo & $\begin{array}{l}\text { Deben tener interés en la temática educativa, que concuerde su profesión con el } \\
\text { ámbito social y en la búsqueda de soluciones gubernamentales y ONGs, que sepa } \\
\text { coordinar grupos amplios y diversos, con una inteligencia kinestésico espacial } \\
\text { relevante. }\end{array}$ \\
\hline $\begin{array}{l}\text { Talleres vinculantes a } \\
\text { la Comunidad } \\
\begin{array}{l}\text { Educativa } \\
\text { Talleres por líneas de } \\
\text { investigación }\end{array}\end{array}$ & $\begin{array}{l}\text { Desarrollar guías de trabajo con mesas que sean motivados con facilitadores } \\
\text { vinculantes a la comunidad padres de familia o representantes legales. }\end{array}$ \\
$\begin{array}{l}\text { Actividades } \\
\text { estratégicas y } \\
\text { propuestas soluciones }\end{array}$ & Según el espíritu de los convenios Marco y específicos. \\
\hline
\end{tabular}




\begin{tabular}{ll}
\hline Diseño del proyecto & $\begin{array}{l}\text { Se plantean el diseño con: introducción, línea base, objetivos, actividades, } \\
\text { presupuesto, cronograma de actividades. Se requiere que se utilice el marco lógico } \\
\text { como técnica de diseño y en el marco de los lineamientos de la Universidad. }\end{array}$ \\
$\begin{array}{l}\text { Recursos de } \\
\text { seguimiento y } \\
\text { monitoreo }\end{array}$ & $\begin{array}{l}\text { Se realizan informes mensuales, matrices de seguimiento y monitoreo, fichas de } \\
\text { evaluación docentes y estudiantes }\end{array}$
\end{tabular}

Elaborado por: MSc Ericka Figueroa Martínez y MSc Lucrecia Resabala

\section{Las competencias docentes como proyección de la Gestión Social del Conocimiento}

El profesional en educación, es el orientador de los proyectos de vida; ello significa que debe asumir con responsabilidad esta función encomendada por la sociedad. Las diferentes IES ${ }^{1}$ deben presentar diferentes alternativas para el desarrollo de la vinculación respectivamente promoviendo el desarrollo de su espíritu de emprendimiento y praxis educativa.

(Figueroa, 2016) "La escuela debe ser el escenario propicio para desarrollar el proyecto de vida que a futuro se promulgará la empatía no sólo del mundo laboral sino social"

El desarrollo de competencias de los estudiantes se estructura con una firme planificación de antecedentes, introducción, objetivos, actividades, presupuesto, seguimiento y evaluación del proyecto.

(Noro, 2010)“La escuela como ámbito, como estructura edilicia, como espacio común público y compartido de las actividades educativas se convierte en un escenario en que los diferentes actores representan diferentes papeles"

Los proyectos de las facultades entregados a la Gestión Social del conocimiento realizado en un sondeo a estudiantes de la Carrera de Mercadotecnia arrojaron las siguientes expectativas vinculantes a las necesidades con la comunidad.

El reporte del trabajo:

- Consultorio Jurídico

- Liga Deportiva Universitaria

- Policlínico Universitario

- Radio Stereo Comunitario

- Escuela de Música y Arte

- Desarrollo de promoción cultural ( raíces culturales)

- Revista Comunitaria

- Centro de Innovación Tecnológica

Los proyectos debe estar realizado por las universidades académicas deben realizar investigaciones, diseñar propuestas de acuerdo a las demandas sociales de cada una de las carreras y ejecutar la función de vinculación con la colectividad sin olvidar, monitorear y evaluar

\footnotetext{
${ }^{1}$ Instituciones de Educación Superior
} 
los procesos, pero deben realizar un verdadero sondeo del proceso, para revisar la pertinencia de los mismos.

\section{Conclusiones}

El organismo de Gestor Social del Conocimiento reconoce la importancia y desarrollo de evaluar el perfil de los supervisores, según la glocalidad esto deben que responder a la necesidad de cultura, etnia, lingüística y constructo social del proyecto de vinculación al cual tributan.

La permanencia del proyecto debe ser evaluado según su planificación y gerencia dentro de los organismos de verificación de la institución anfitriona.

\section{Bibliografía}

Bordieau. (2000). La objetivación participante. París: actes de rechenhe en suencier sociales.

Caicedo, c. G. (2014). El proceso extensionista universitario como vía para la pertinencia en la formacion del futuro profesional. Espamciencia, 17-24.

Ces. (2015). Plan de excelencia ug. Quito: senescyt.

Figueroa, e. (2016). Proyecto de vida. Ciencia y tecnología, 11-23.

Internacional, o. (15 de enero de 2016). Tecnicas participativas. Ciudadania y medio ambiente sostenible. Guadalajara: cimas.

Morín, e. (2011). La vía para el futuro de la humanidad. Barcelona: paidos.

Noro, e. (2010). Actitudes y valores puerta de entrada de una escuela significativa. Rosario: biblioteca docente.

Perez. (2010). Conocimiento sobre ekl proceso extensionista universitario en la formación del protagonismo del futuro profesional. Revista de estudios interdisciplinario en ciencias sociales, 11-30.

Ponce. (2006). La matriz foda: una alternativa para realizar diagnósticos y determinar estrategias de intervención en las organizaciones productivas y sociales.

Porter. (1998). Técnicas para el análisis de los sectores industriales. México: editorial cecsa.

Ramirez. (2012). Dimensiones constituidas y ejes estructurales de la ciudadnia. Mexico: unam.

Sodexo. (2015). Caracterización del gestor social del conocimiento. Lima: república del perú.

Talancón, h. P. (septiembre de 2006). Contribuciones a la economía. Obtenido de http://www.eumed.net/ce/2006/hpt-foda.htm 
Ug. (2016). Competencias básicas de la universidad de guayaquil. Quito.

Ug. (2016). Funciones sociales del gestor social del conocimiento. Quito. 